\title{
Change detection in the flicker paradigm: The role of fixation position within the scene
}

\author{
ANDREW HOLLINGWORTH, GARY SCHROCK, and JOHN M. HENDERSON \\ Michigan State University, East Lansing, Michigan
}

\begin{abstract}
Eye movements were monitored while participants performed a change detection task with images of natural scenes. An initial and a modified scene image were displayed in alternation, separated by a blank interval (flicker paradigm). In the modified image, a single target object was changed either by deleting that object from the scene or by rotating that object $90^{\circ}$ in depth. In Experiment 1 , fixation position at detection was more likely to be in the target object region than in any other region of the scene. In Experiment 2, participants detected scene changes more accurately, with fewer false alarms, and more quickly when allowed to move their eyes in the scene than when required to maintain central fixation. These data suggest a major role for fixation position in the detection of changes to natural scenes across discrete views.
\end{abstract}

When we view natural scenes, our impression is that the mind constructs something like an internal photographic image of the entire field of view. However, investigations of visual short-term memory (VSTM) across saccadic eye movements suggest that the visual information retained from one eye fixation to the next is abstracted away from low-level sensory stimulation and that VSTM preserves information from only a small portion of the visual field (Currie, McConkie, Carlson-Radvansky, \& Irwin, 2000; Grimes, 1996; Henderson \& Hollingworth, 1999b; Henderson, Hollingworth, \& Subramanian, 1999; McConkie \& Currie, 1996). These researchers have employed change detection paradigms in which a change to a scene is completed during a saccadic eye movement, when visual encoding is suppressed. Participants are often surprisingly insensitive to quite significant changes, suggesting that a visually veridical, global image of the scene is not constructed and retained across eye movements.

To determine whether such insensitivity to scene changes is a general property of visual perception, Rensink, O'Regan, and Clark (1997) developed a flicker paradigm that simulated the visual events caused by moving the eyes but that did not depend on eye movements to initiate scene changes. A series of images was presented that alternated

This research was supported by an NSF Graduate Research Fellowship to A.H. and NSF Grants SBR 9617274 and ECS 9873531 to J.M.H. A brief report of this work was presented at the 39th Annual Meeting of the Psychonomic Society in Dallas, November 1998. We thank Ron Rensink and Steffen Werner for helpful comments on an earlier version of this paper, Ben Swets and Robin Revette for running the experiment sessions, and the following graphic artists for donating 3-D models used to create some of the experimental stimuli: Pal Karlsen, Thomas Sellner, Marco Iozzi, Mette Olsen, Heinz Schuller, Marcel Houtveen, Nezih Kanbur, and Tarik Dzevdetbegovic. Please address correspondence to A. Hollingworth, Department of Psychology, Yale University, Box 208205, New Haven, CT 06520-8205 (e-mail: andrew. hollingworth@yale.edu). between an initial image and a modified image of the same scene. A blank interval was presented between scenes, corresponding to the visual suppression caused by a saccadic eye movement. As in the saccade-contingentchange studies, participants often failed to detect some fairly large changes. This phenomenon of poor change detection to visual scenes-across eye movements and artificial disruptions - has come to be termed change blindness (Rensink et al., 1997; see Simons \& Levin, 1997, for a review).

A prominent view in the change blindness literature is that change detection in the flicker paradigm depends on the allocation of visual attention to the changing region (Rensink, 2000a; Rensink et al., 1997; Simons \& Levin, 1997; Wolfe, 1999), where visual attention is defined as an internal mechanism for selecting certain visual codes for further processing at the expense of other visual codes. In this view, visual attention serves to maintain a representation of an attended object in VSTM across the blank interval, enabling comparison and change detection. In contrast, unattended scene information decays rapidly upon scene offset and is overwritten by subsequent visual encoding. Thus, if the changing and attended regions do not overlap, the change will not be detected.

In three studies researchers have sought to provide evidence that the detection of changes to scenes depends on the allocation of visual attention to the changing region. Rensink et al. (1997) found that changes to areas of a scene rated to be of "central interest" were detected more quickly than changes to "marginal interest" areas. They argued that this difference in detection performance was due to the fact that central interest areas were preferentially selected by visual attention. This conclusion, however, is problematic, because Rensink et al. had no means of determining where visual attention was allocated in the scenes independently of the detection of changes. Thus, they could not know whether central interest regions were indeed preferentially selected by visual attention. 
Two more recent studies provide stronger evidence in favor of the attention hypothesis. Scholl (2000) used a peripheral cuing manipulation to study the role of visual attention in the flicker paradigm. During each trial, an array of line drawings of objects was presented, and a single object was changed periodically between two array images. This object could be either cued by means of an abrupt onset or color singleton-manipulations that have been demonstrated to capture visual attention (see, e.g., Jonides, 1981; Theeuwes, 1991)—or uncued. Consistent with the possibility that visual attention is critical to the detection of scene change in the flicker paradigm, changes to cued objects were detected faster than changes to uncued objects. In addition, Rensink (2000b) employed a visual search variant of the flicker paradigm to study the role of visual attention in change detection. Rensink found that search times for changing conjunction targets were linearly related to stimulus set size and that search slopes in the target-present trials were approximately half those in target-absent trials. These results suggest a serial, selfterminating search, consistent with explanations of search performance in terms of visual attention.

The data on visual attention and change detection in the flicker paradigm are consistent with the broad claims of the attention hypothesis. To this point, however, the attention hypothesis has not addressed another important factor governing the selection of visual information: the overt movement of the eyes. The distinction between eye movements and visual attention is important, because these two mechanisms of visual selection can be dissociated experimentally (e.g., Posner, Snyder, \& Davidson, 1980) and diverge on a number of selection and orienting characteristics. For example, the spatial extent of a visually attended region can vary dynamically according to the visual characteristics of the display and other task constraints (Eriksen \& St. James, 1986; Henderson, 1991), whereas the physical structure of the retina determines the region of enhanced visual processing afforded by fixation.

Eye movements have not been monitored or controlled in any of the flicker paradigm studies implicating visual attention in change detection. Thus, we cannot know whether the results of these studies reflect the orienting of the eyes rather than the orienting of visual attention. In addition, evidence that changes to "central interest" regions are detected faster than changes to "marginal interest" regions is consistent with a large body of research demonstrating that more informative regions of a scene are fixated earlier, longer, and more often than less informative regions (see Henderson \& Hollingworth, 1999a, for a review). Rensink et al.'s (1997) results could be explained simply by the fact that participants tend to notice changes to regions they are currently fixating. Further reason to expect that fixation position influences change detection in the flicker paradigm comes from the literature on change detection across saccadic eye movements (Currie et al., 2000; Henderson \& Hollingworth, 1999b;
Hollingworth, Williams, \& Henderson, in press; McConkie \& Currie, 1996; O'Regan, Deubel, Clark, \& Rensink, 2000; see Henderson \& Hollingworth, in press, for a review). For example, Henderson and Hollingworth (1999b) found better change detection performance when the changing object was fixated either directly before the change or directly after the change in comparison with when that object was not fixated in either the initial or the modified scene.

The purpose of the present study, then, was to determine whether fixation position influences the detection of scene changes in the flicker paradigm. In Experiment 1, we monitored eye movements while participants performed the flicker task, in order to determine whether there is a relationship between fixation position and change detection. In Experiment 2, we sought to determine whether fixation position plays a significant causal role in change detection independently of the allocation of visual attention. Fixation position was dissociated from the orienting of visual attention by either requiring participants to maintain central fixation or allowing them to move their eyes freely, as in Experiment 1.

\section{EXPERIMENT 1}

To establish whether there is a relationship between fixation position and change detection, eye movements were monitored as participants performed the flicker task. Scene stimuli were computer-rendered color images of real-world environments. An initial and a modified version of a scene were presented in an alternating sequence, each separated by a brief blank interval. There were two change conditions. In the deletion-addition condition, a target object in the initial scene was deleted from the modified scene. Across repetitions of the change cycle, this object would periodically disappear from the scene and then reappear. In the rotation condition, the target object in the initial scene was rotated $90^{\circ}$ around its vertical axis in the modified scene. Across repetitions of the change cycle, this object would periodically rotate between two orientations in depth. If fixation position is related to change detection in the flicker paradigm, fixation position when a change is detected should be close to or should coincide with the position of the changing object.

\section{Method}

Participants. Eight Michigan State University undergraduate students participated in the experiment for course credit. All participants had normal vision and were naive with respect to the hypotheses under investigation.

Stimuli. Thirty-five scene images were computer rendered from 3-D wire-frame models using 3-D graphics software (3D Studio Max). Each model depicted a typical, human-scaled environment (e.g., "attic" or "art gallery"). To create each initial scene image, a target object was chosen within the model, and the scene was rendered so that this target object did not coincide with the initial experimenterdetermined fixation position. To create the deletion-addition condition scene images, the scene was re-rendered after the target object had been deleted from the model. To create the rotation condition 


\section{Initial Image}

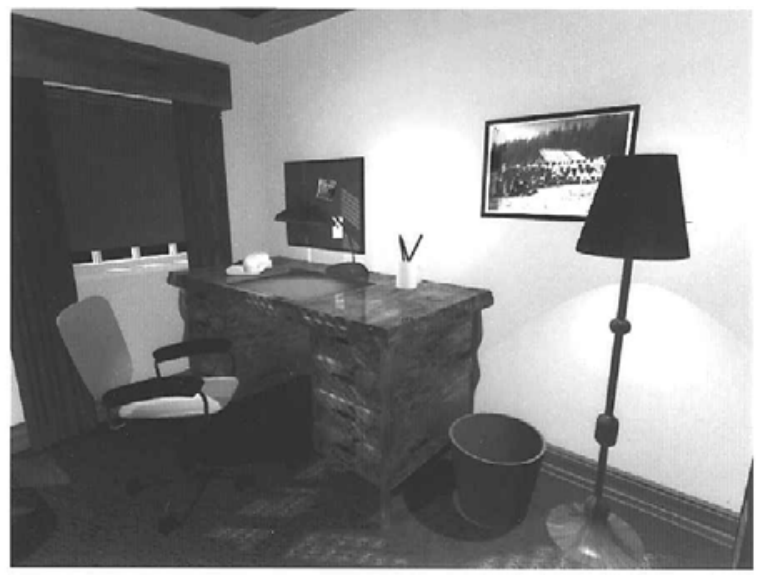

\section{Modified Image: Deletion}

\section{Modified Image: Rotation}
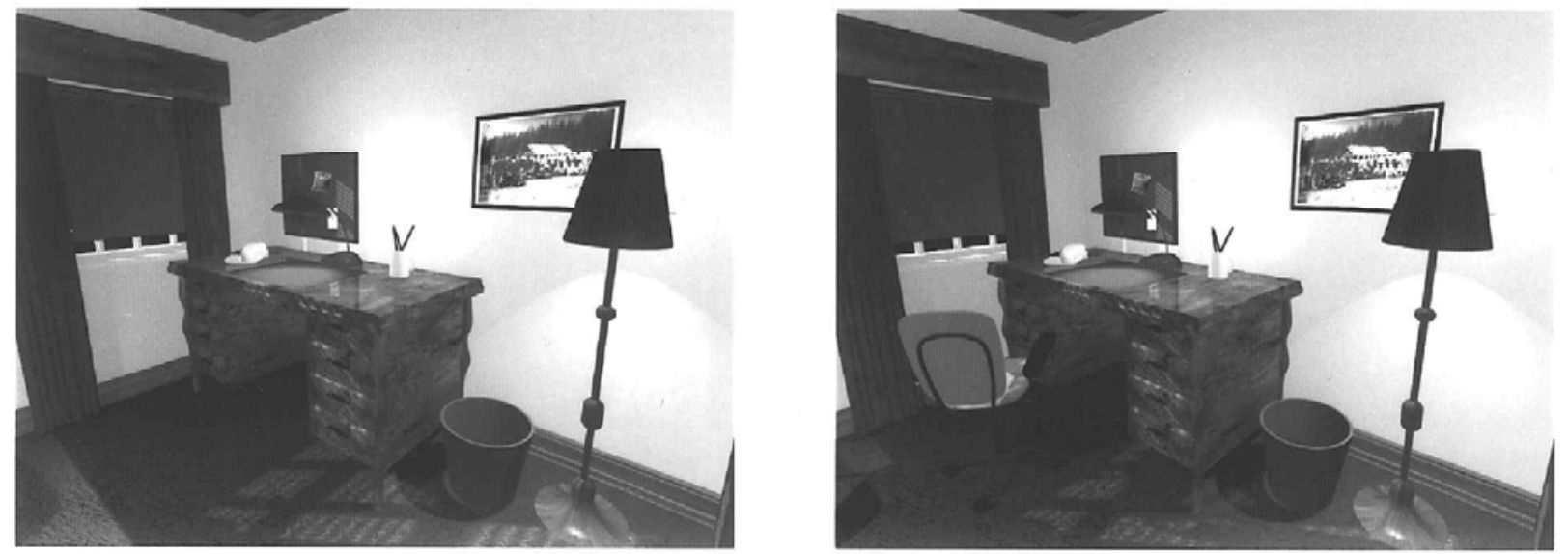

Figure 1. An example of a stimulus scene and the change conditions (experimental images appeared in color). The desk chair is the target object. This object was deleted from the scene between the initial and modified images in the deletion-addition condition. This object was rotated $90^{\circ}$ around its vertical axis between the initial and modified images in the rotation condition.

scene images, the scene was re-rendered after the target object had been rotated $90^{\circ}$ around its vertical axis. Five additional scene images were created from different wire-frame models to serve in the catch trials. All scene images subtended $15.8^{\circ} \times 11.9^{\circ}$ visual angle at a viewing distance of $1.13 \mathrm{~m}$. Target objects subtended $2.43^{\circ}$ on the average along the longest dimension in the picture plane. Figure 1 shows a sample stimulus scene, along with the two change conditions. The blank screen appearing between scene images subtended the same visual angle as did the scenes and was set to a uniform gray at approximately the same luminance as that of the scenes.

Procedure. The participants were instructed that their eye movements would be monitored while they viewed images of naturalistic scenes on a computer monitor. These images would flicker on and off during a trial, and on most trials, a change would occur periodically to a single object in the scene. On some trials, however, no change would occur. Participants were instructed to press a response button immediately upon detecting a change in the scene. The two types of possible changes were described.
A trial consisted of the following events. First, a fixation screen was shown. When the participant fixated a central box in this screen, the experimenter started the trial. The initial (A) and modified $\left(\mathrm{A}^{\prime}\right)$ scenes were displayed for $250 \mathrm{msec}$ each in the following sequence: $\mathrm{A}, \mathrm{A}, \mathrm{A}^{\prime}, \mathrm{A}^{\prime}$. A blank (gray) screen was presented after each scene for $80 \mathrm{msec}$. Thus, a change occurred every $660 \mathrm{msec}$ during a trial. In the change trials, the sequence was repeated for $40 \mathrm{sec}$ or until the participant pressed the response button. In the catch trials, the sequence was repeated for $20 \mathrm{sec}$ or until the participant pressed the response button. ${ }^{1}$ In previous flicker studies, participants have been required to report the identity and location of the changed object. In the present study, we were interested in the position of the eyes at the time when a change was initially detected. Thus, we instructed participants to respond immediately upon detecting a change, and we did not require them to report the nature or location of the change. No-change catch trials were used to assess whether the participants followed instructions to respond only after having detected a change. 
Following review of the instructions, the participants took part in two practice trials, one in the rotation condition and one in the deletion-addition condition. The practice scenes were not included in the experimental session. After the practice session, the experimenter calibrated the eyetracker by having the participants fixate four markers at the centers of the top, bottom, left, and right sides of the display. Calibration was considered accurate if the computer's estimate of the current fixation position was within $\pm 5^{\prime}$ of arc of each marker. The participant then completed the experimental session. Calibration was checked every three to four trials, and the eyetracker was recalibrated whenever necessary.

In the experimental session, each participant saw all 35 scenes, 17 in one change condition and 18 in the other. ${ }^{2}$ The assignment of items to conditions was counterbalance $\mathrm{d}$ between participant groups. Across participants, each scene appeared in each change condition an equal number of times. In addition to the 35 change trials, 5 catch trials were included in which no change occurred. The scenes used in the catch trials did not appear in either of the change conditions. Trial order was determined randomly for each participant. The entire experiment lasted approximately $35 \mathrm{~min}$.

Apparatus. The stimuli were displayed at a resolution of $800 \times$ 600 pixels $\times 256$ colors. The display monitor refresh rate was set at $143 \mathrm{~Hz}$. The room was dimly illuminated by an indirect, low-intensity light source.

Eye movements were monitored using a Generation 5.5 Stanford Research Institute Dual Purkinje Image Eyetracker (Crane \& Steele, 1985). A bite bar and forehead rest were used to maintain the participant's viewing position. The position of the right eye was tracked, though viewing was binocular. Eye position was sampled at rate of better than $1000 \mathrm{~Hz}$. Buttonpresses were collected using a button panel connected to a dedicated input-output (I/O) card. The eyetracker, display monitor, and I/O card were interfaced with a $90 \mathrm{MHz}$, Pentium-based microcomputer. The computer controlled the experiment and maintained a complete record of time and eye position values over the course of each trial.

\section{Results and Discussion}

In this section, we will first report general measures of detection performance. Then we will report eye movement analyses concerning the relationship between detection and fixation position. Where appropriate, two analyses of variance (ANOVAs) were conducted, one treating participant as a random effect $\left(F_{1}\right)$ and one treating scene item as a random effect $\left(F_{2}\right)$. Reported means were derived from analyses treating participant as a random effect.

Detection percentage. For the change trials, participants detected a change within $40 \mathrm{sec}$ on $98.6 \%$ of the trials in the deletion-addition condition and $97.8 \%$ of the trials in the rotation condition. These percentages were not reliably different $(F \mathrm{~s}<1)$. There were no false alarms for any participant in the no-change catch trials.

Detection latency. Detection latency was calculated for each correct detection trial in the change conditions. One trial was eliminated because the participant responded before the first scene change occurred. This response can be considered a false alarm; however, the fact that there were no false alarms in the catch trials suggests that this response was anomalous.

Detection latency was shorter for deletion additions $(2.76 \mathrm{sec})$ than for rotations $(4.37 \mathrm{sec})\left[F_{1}(1,7)=13.90\right.$, $M S_{\mathrm{e}}=.7487, p<.01 ; F_{2}(1,34)=11.37, M S_{\mathrm{e}}=4.516, p<$ $.005]$. This result is not particularly surprising given that deletion additions tend to be larger visual disruptions than rotations. In addition, deletion additions constitute a semantic change in the scene, whereas rotations typically do not. Overall, detection latency was shorter in this experiment than in other flicker studies (e.g., Rensink et al., 1997). Our shorter latencies were likely due to that fact that changes occurred to whole, discrete objects rather than to parts of objects or single properties of objects (e.g., color), as in some of the Rensink et al. changes. There was, however, a great deal of variability in the mean detection latency for different scene items. The easiest detection, a deletion-addition change, required only $1.1 \mathrm{sec}$ on the average (less than two change cycles), and the most difficult detection, a rotation change, required $12.9 \mathrm{sec}$ on the average (more than 19 change cycles).

Fixation position at time of detection. Eye movement data files were first analyzed to identify periods of fixation and of saccadic movement (see Henderson, McClure, Pierce, \& Schrock, 1997). Fixations lasting less than $90 \mathrm{msec}$ were eliminated as outliers. Due to the fact that fixation durations were sometimes quite long in this experiment, we set a conservative upper fixation duration cutoff of 3,550 msec. Figure 2 shows the distribution of fixations for each participant on one scene in the deletion-addition and rotation conditions.

As an initial test of the role of eye movements in the flicker paradigm, we examined for each correct detection trial whether the participant's eye left a $3^{\circ} \times 3^{\circ}$ region of the scene centered on the initial fixation point. If change detection depends strongly on fixation position, we should find that participants rarely confined their gaze to this central region. Supporting this prediction, participants' eyes remained in the central region on only $7.7 \%$ of the trials. ${ }^{3}$ This result suggests that participants do not typically detect changes in the flicker paradigm by covertly monitoring extrafoveal regions of the scene. Instead, they actively fixated potential change targets, as is evident in Figure 2.

To assess the relationship between eye position and change detection, we analyzed the position of the eyes when the participant pressed the response button. It is possible that the position of the eyes at the time of response might not have always reflected eye position at the time that the change was initially detected. Thus, we also computed the position of the eyes when the final change in the scene occurred prior to response. Four scoring regions were defined for each target object in each scene. The target object region was defined by creating the smallest circle that could encompass the target object. The foveal region was defined as a $1^{\circ}$-wide ring surrounding the target object region. Thus, if the eyes fixated the edge of the foveal region farthest from the target object, the nearest contours of that object would generally fall in foveal vision. By the same logic, the parafoveal region was defined as a $2.5^{\circ}$-wide ring surrounding the target object and foveal regions. Finally, the peripheral region was defined as any part of the scene not included in the three regions described above. 

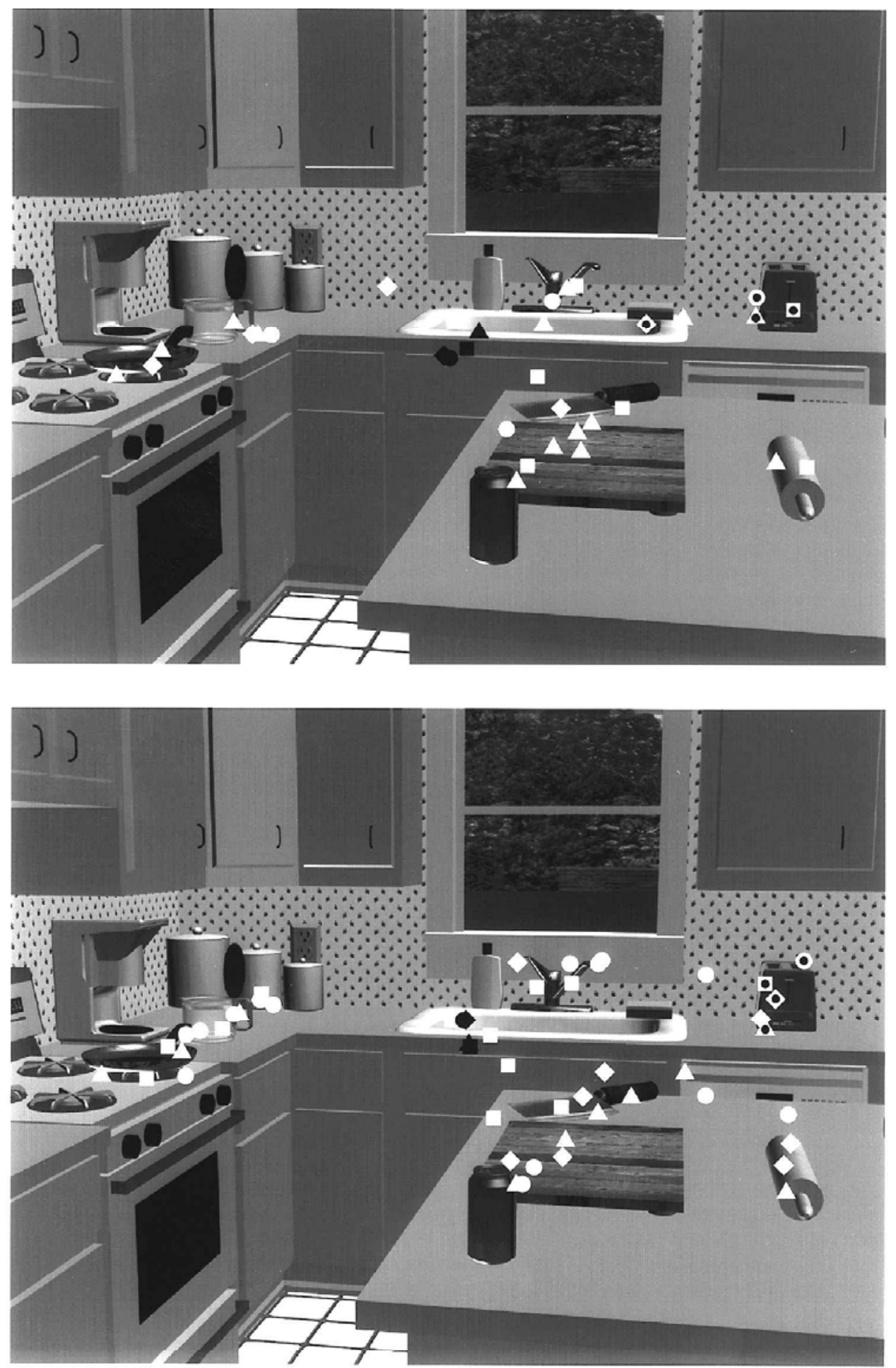

Figure 2. Plot of all fixations for all participants on a stimulus scene. The fixations of the 4 participants who saw this scene in the deletion-addition condition (top panel) are distinguished by four different fixation symbols (circle, square, diamond, and triangle). The same four symbols distinguish the fixations of the 4 participants who saw this scene in the rotation condition (bottom panel). Black symbols represent the initial, experimenter-determined fixation on the scene. White symbols represent subsequent fixations on the scene. White symbols with a black dot indicate fixation position when the participant pressed the response button. The toaster was the target object. Note that this figure shows fixation patterns superimposed on the initial image only. In the actual trials, this image was replaced periodically with a modified image in which the toaster was either deleted from the scene or rotated $90^{\circ}$ around its vertical axis. 
Table 1

Percentage of Fixations on the Scene in Each of the Four Scoring Regions at Response and at Final Change, Experiment 1

Percentage in Each Region

\begin{tabular}{lcrcc} 
Change Condition & Target Object & Foveal & Parafoveal & Peripheral \\
\cline { 2 - 5 } Deletion-Addition & & & & \\
At response & 63.9 & 10.2 & 9.9 & 16.1 \\
At final change & 47.5 & 9.2 & 16.6 & 26.8 \\
Rotation & & & & \\
At response & 85.0 & 6.8 & 3.0 & 5.2 \\
At final change & 77.3 & 8.4 & 4.5 & 9.8 \\
\hline
\end{tabular}

The proportion of fixations falling in each region, both at response and at final change, is presented in Table 1 . The large majority of fixations at response were within the target object region (74.5\%), a result that is particularly striking given that the target object region occupied a relatively small portion of each scene. The peripheral region, on the other hand, accounted for only $5.9 \%$ of fixations. In addition, more fixations at response fell on the target object in the rotation condition $(85.0 \%)$ than in the deletion-addition condition $(63.9 \%)\left[F_{1}(1,7)=14.53, M S_{\mathrm{e}}=\right.$ $\left.122.5, p<.01 ; F_{2}(1,34)=11.76, M S_{\mathrm{e}}=745.1, p<.005\right]$. Presumably, participants required foveal information more often when the change was more difficult to detect.

If we turn next to fixation position at the time of the final change, there is some indication that target objects were fixated less often at the final change than at response, particularly in the deletion-addition condition. However, the patterns of data in the two analyses are quite similar. The majority of fixations at the final change was in the target object region $(62.4 \%)$, and the target object and foveal regions together accounted for $71.2 \%$ of fixations. As in the fixation position at response analysis, more fixations at final change fell in the target object region in the rotation condition $(77.3 \%)$ compared with the deletion-addition condition $(47.5 \%)\left[F_{1}(1,7)=25.78\right.$, $M S_{\mathrm{e}}=138.1, p<.005 ; F_{2}(1,34)=13.00, M S_{\mathrm{e}}=1,250$, $p<.005]$. In summary, the position of the eyes both at response and at final change was more likely to be in the target object region than in any other region of the scene, and this effect was more pronounced for rotation changes than for deletion-addition changes.

The relationship between detection and eye position raises an additionalquestion. Was fixation of the target object independent of the change occurring in the scene, or were fixation patterns influenced by scene changes? In particular, we were interested in whether the eyes were attracted to regions of change. To investigate the question, we examined the elapsed time from the beginning of the trial to the first fixation on the target object region for the deletion-addition condition and the rotation condition. This analysis was conditional on target fixation. The mean elapsed time was $2.10 \mathrm{sec}$ in the deletion-addition condition and $2.89 \mathrm{sec}$ in the rotation condition. This difference was reliable by items $\left[F_{2}(1,33)=6.46, M S_{\mathrm{e}}=1.228, p<\right.$ $.05]$ but not by participants $\left[F_{1}(1,7)=2.49, M S_{\mathrm{e}}=1.007\right.$, $p=.16] .{ }^{4}$ Thus, the present data provide some indication that eye movement patterns were sensitive to the changes occurring in the scenes.

To further assess whether eye movement patterns were sensitive to changes in the scenes, we conducted a control experiment in which no change occurred on $50 \%$ of the trials. ${ }^{5}$ This control experiment allowed us to compare the elapsed time to the first fixation on the target region when a change was occurring to this object with that when no change was occurring. Because the number of observations in each of the change conditions in this experiment was small, we collapsed across change type to calculate the elapsed time measure for changing objects. The elapsed time to the first fixation on the target object was $3.50 \mathrm{sec}$ in the no change condition and $2.76 \mathrm{sec}$ in the change condition. This difference was not reliable by participants $\left[F_{1}(1,7)=2.25, M S_{\mathrm{e}}=.9953, p=.18\right]$, but it did approach reliability by items $\left[F_{2}(1,32)=2.72, M S_{\mathrm{e}}=4.340, p=.11\right]$. As an additional test, we compared the mean elapsed time in the no-change condition to the two change conditions of the main experiment. The elapsed time to the first fixation on the target object was reliably longer for the no-change control than for the deletion-addition condition of the main experiment $\left[F_{1}(1,14)=15.7, M S_{\mathrm{e}}=.5036, p<\right.$ $\left..005 ; F_{2}(1,33)=24.4, M S_{\mathrm{e}}=1.959, p<.001\right]$. The difference between the no-change control and the rotation condition of the main experiment was reliable by items but not by participants $\left[F_{1}(1,14)=1.39, M S_{\mathrm{e}}=1.091, p=\right.$ $\left..26 ; F_{2}(1,34)=9.23, M S_{\mathrm{e}}=1.752, p<.005\right]$. Thus, there is some evidence that the eyes are directed more quickly to an object when it is changing than when it is not.

\section{EXPERIMENT 2}

The results of Experiment 1 indicate a strong relationship between fixation position and change detection in the flicker paradigm. However, fixation position and visual attention tend to be spatially and functionally linked during normal viewing: Visual attention is preferentially allocated to the current fixation position, and the covert orienting of visual attention precedes the eyes to the next fixation target (see Henderson, 1996). Therefore, the relationship between fixation position and change detection in Experiment 1 might not have been caused by fixation position per se but instead by the preferential allocation of visual attention to fixated objects and to the next saccade target. Thus, the results of Experiment 1 do not rule out the possibility that visual attention is solely functional in detecting changes.

In Experiment 2, we included a manipulation to dissociate the orienting of visual attention from fixation position. In one session of the experiment, participants were required to maintain central fixation (no-movement condition). In a different session of the experiment, participants were free to move their eyes as in Experiment 1 (movement condition). In each session, trials were evenly distributed between three change conditions (additiondeletion, rotation, and no change). If fixation position 
Table 2

Percentage Correct and Detection Latency as a Function of Eye Movement Condition and Change Type, Experiment 2

\begin{tabular}{lccccccc}
\hline & \multicolumn{3}{c}{ Movement } & & \multicolumn{3}{c}{ No Movement } \\
\cline { 2 - 4 } \cline { 6 - 8 } Measure & Addition-Deletion & Rotation & No Change & & Addition-Deletion & Rotation & No Change \\
\hline \% Correct & 100.0 & 98.8 & 100.0 & & 96.0 & 86.0 & 81.9 \\
Latency $(\mathrm{sec})$ & 2.93 & 4.47 & - & & 5.05 & 7.64 & - \\
\hline
\end{tabular}

Note-The false alarm rate is 100 minus percent correct in the no-change condition.

plays a significant causal role in change detection independently of the orienting of visual attention, detection performance should be reliably higher when regions of change can be attended and fixated (movement condition) than when they can be attended covertly but not fixated (no-movement condition). In addition, if target fixation is the sole determinant of detection performance, then the task should be exceedingly difficult or impossible in the no-movement condition.

\section{Method}

Participants. Twelve Michigan State University undergraduate students participated in the experiment for course credit. All participants were naive with respect to the hypotheses under investigation and had not participated in Experiment 1.

Stimuli. A total of 42 scene stimuli were used: the original 35 from Experiment 1, and 7 new scenes created in the same manner as the original 35 but from different 3-D models.

Procedure. The procedure was the same as that of Experiment 1, with the following modifications. After describing the task and the types of possible changes, the experimenter informed participants that in one session of the experiment, they were to keep their eyes focused on the center of the screen throughout the trial, and in the other session, they would be free to move their eyes around the scene.

Following review of the instructions, the participants took part in 6 practice trials, 3 in the movement condition and 3 in the no-movement condition. The 6 practice scenes were not included in the experimental session. In the experimental sessions, each participant saw all 42 scenes, 7 in each of the 6 conditions created by the $2 \times 3$ factorial combination of eye movement condition (movement, no movement) and change type (deletion-addition, rotation, no change). The assignment of items to conditions was counterbalanced between participant groups. Across participants, each scene appeared in each condition an equal number of times. On each trial, the flicker sequence was repeated for $40 \mathrm{sec}$ or until the participant pressed the response button. Trial order was determined randomly for each participant. The entire experiment lasted approximately $40 \mathrm{~min}$.

During the experimental sessions, the experimenter monitored a video image of the right eye. In the no-movement session, the experimenter recorded trials on which the participant made an eye movement. After such trials, the experimenter reminded the participant to maintain fixation at the center of the screen. The experimenter also monitored the participant's eye in the movement session to confirm that the eyes were indeed moving.

Apparatus. The apparatus was the same as in Experiment 1, with the following modifications. First, the stimuli were presented at 15 -bit resolution rather that at 256 -color resolution. Second, eye movement data were not collected with the use of an eyetracker. Eye movements were detected with a video monitor displaying a close-up image of the right eye.

\section{Results and Discussion}

We were interested in three measures of change detection performance: percentage correct detections, per- centage false alarms, and response latency for correct detections. These data are presented in Table 2. Trials on which an eye movement was detected in the no-movement condition were eliminated from all analyses. These trials accounted for $4.2 \%$ of the data. In a preliminary analysis, we examined the counterbalancing factor of eye movement session order for each of the measures of interest. Because session order produced neither a reliable main effect nor an interaction with any other factor, all subsequent analyses collapsed across session order.

Percentage correct detections. Percentage correct data were analyzed as a $2 \times 2$ factorial: eye movement condition (movement, no movement) $\times$ change condition (deletion-addition, rotation). First, there was a reliable effect of eye movement condition $\left[F_{1}(1,11)=8.28, M S_{\mathrm{e}}=\right.$ $\left.101.7, p<.05 ; F_{2}(1,41)=11.41, M S_{\mathrm{e}}=333.9, p<.005\right]$. Percentage correct in the movement condition (99.4\%) was higher than that in the no-movement condition (91.0\%). Second, there was an effect of change condition that was reliable by participants and approached reliability by items $\left[F_{1}(1,11)=4.76, M S_{\mathrm{e}}=78.99, p<.05 ; F_{2}(1,41)=2.78\right.$, $\left.M S_{\mathrm{e}}=342.6, p=.10\right]$, with higher detection performance in the deletion condition (98.0\%) than in the rotation condition $(92.4 \%)$. These factors did not interact $\left[F_{1}(1,11)=\right.$ $2.53, M S_{\mathrm{e}}=92.01, p=.14 ; F_{2}(1,41)=1.84, M S_{\mathrm{e}}=291.8$, $p=.18]$.

Percentage false alarms. The percentage of false alarms was reliably higher in the no-movement condition than in the movement condition $\left[F_{1}(1,11)=14.65, M S_{\mathrm{e}}=\right.$ $134.5, p<.005 ; F_{2}(1,41)=18.38, M S_{\mathrm{e}}=273.7, p<$ $.001]$. There were no false alarms in the movement condition but $18.1 \%$ false alarms in the no-movement condition.

Detection latency. First, there was a reliable effect of eye movement condition $\left[F_{1}(1,11)=10.80, M S_{\mathrm{e}}=7.766\right.$, $\left.p<.01 ; F_{2}(1,41)=30.95, M S_{\mathrm{e}}=10.13, p<.001\right]$. Detection latency in the movement condition $(3.70 \mathrm{sec})$ was shorter than that in the no-movement condition $(6.34 \mathrm{sec})$. Second, there was a reliable effect of change condition $\left[F_{1}(1,11)=13.85, M S_{\mathrm{e}}=3.707, p<.005 ; F_{2}(1,41)=\right.$ $\left.12.99 ; M S_{\mathrm{e}}=12.52, p<.005\right]$, with shorter detection latency in the deletion condition $(3.99 \mathrm{sec})$ than in the rotation condition $(6.06 \mathrm{sec})$. These factors did not interact $\left(F_{1}<1.25 ; F_{2}<1\right)$.

The results of Experiment 2 indicate that fixation position plays a significant causal role in the detection of scene changes in the flicker paradigm. Percentage correct detections were higher, percentage false alarms were lower, and detection latency was shorter when participants 
could fixate changing objects than when they could covertly attend but could not fixate those objects. The difference in detection latency was particularly striking, with latency in the no-movement condition approximately $70 \%$ higher than that in the movement condition. Although the task was much more difficult when participants maintained central fixation, it was not impossible, however. The percentage of correct detections in the no-movement condition was near $90 \%$. Although this measure may overestimate detection performance given that there was a significant number of false alarms (18.1\%), sensitivity to changes in the no-movement condition was still well above chance. Thus, it does not appear that fixation position is the sole determinant of change detection performance in the flicker paradigm.

\section{GENERAL DISCUSSION}

In this study, we investigated whether fixation position influences the detection of scene changes in the flicker paradigm. In Experiment 1, we examined the relationship between fixation position and change detection by monitoring eye position during the flicker task. When a change was detected, the eyes were more likely to be fixating the changing object than any other region of the scene, suggesting a close link between fixation position and detection. In Experiment 2, we sought to dissociate effects of fixation position from those potentially attributable to the orienting of visual attention. Participants either were allowed to move their eyes freely (movement condition) or were required to maintain central fixation (no-movement condition). Percentage correct detections was higher, percentage false alarms was lower, and detection latency was shorter in the movement condition, when changing objects could be attended and fixated, compared with the no-movement condition, when changing objects could be covertly attended but could not be fixated.

The results indicate a significant causal role for fixation position in the maintenance of information across discrete views of a scene, leading to the detection of changes. Fixating the changing object appeared particularly important for the detection of more difficult changes in the rotation condition. Given that, on the average, our changes were detected much more quickly than in the original flicker study (Rensink et al., 1997), it is likely that our data underestimate the extent to which change detection was dependent on target fixation in that earlier study. Thus, in order to understand the nature of the internal representation formed during scene viewing and the means by which scene changes are detected, theories of scene perception will need to account for eye movement behavior, including such factors as how soon, how often, and how long the changing region is fixated.

In addition, these data have implications for the hypothesis that change detection in the flicker paradigm depends on the allocation of visual attention to the changing region (Rensink, 2000a; Rensink et al., 1997; Wolfe, 1999). First, the results of Experiment 2 demonstrate that the allocation of visual attention cannot be the sole determinant of detection performance in the flicker paradigm, because performance was markedly reduced when participants could covertly attend, but could not fixate, the changing object. Second, the results from this study weaken some of the existing experimental evidence supporting the attention hypothesis. Studies implicating visual attention in the detection of changes in the flicker paradigm have not monitored or restricted eye movements (Rensink, 2000b; Rensink et al., 1997; Scholl, 2000). Evidence that fixation position plays a significant causal role in change detection supports the possibility that detection performance in these studies was governed not by the orienting of visual attention but by the overt movement of the eyes. If researchers seek to investigate the role of visual attention in change detection, fixation position will need to be controlled.

It is important to note that although fixation position appears to be a significant factor governing change detection in the flicker paradigm, it is clearly not the only factor. Change detection when participants were not able to move their eyes (the no-movement condition of Experiment 2) was impaired but was not impossible (see also Hollingworth \& Henderson, 2000). In addition, viewing a changing object in foveal or near-foveal vision may not always be sufficient for one to detect a change (Henderson \& Hollingworth, 1999b; O'Regan et al., 2000). One possible explanation for the ability to detect changes in the no-movement condition of Experiment 2 is that detection was governed by the covert orientation of visual attention. However, because we had no way to determine where visual attention was allocated in the scenes, Experiment 2 cannot provide evidence to test this hypothesis.

One final result deserves discussion. In Experiment 1, fixation patterns on the scenes did not appear to be independent of the changes occurring in the scenes. For trials on which the target object was fixated, the eyes reached this object sooner in the deletion-addition condition than in the rotation condition and sooner in both change conditions than in the no-change control condition. One potential explanation for this effect is that participants sometimes gained partial information that a change had occurred (either consciously or unconsciously) that did not surpass a criterion amount of information necessary to trigger response but that nonetheless allowed them to direct their eyes to a candidate area of the scene where the change may have occurred. This explanation is consistent with other studies in which certain changes were not explicitly reported but implicit measures of detection indicated that a change had been registered (FernandezDuque \& Thornton, 2000; Hayhoe, Bensinger, \& Ballard, 1998; Williams \& Simons, 2000). In particular, Hayhoe et al. (1998) found that for changes not detected explicitly, fixation durations on the changed object were longer than when no change occurred. In our study, subthreshold change-detectioninformation may have served as an input to the occulomotor system, causing the eyes to be directed to areas of the scene where the change was occurring. 


\section{REFERENCES}

Crane, H. D., \& Steele, C. M. (1985). Generation-V dual-Purkinjeimage eyetracker. Applied Optics, 24, 527-537.

Currie, C. B., McConkie, G. W., Carlson-Radvansky, L. A., \& IRWIN, D. E. (2000). The role of the saccade target object in the perception of a visually stable world. Perception \& Psychophysics, 62, 673-683.

ERIKSEN, C. W., \& ST. JAmes, J. D. (1986).Visual attention within and around the field of focal attention: A zoom lens model. Perception \& Psychophysics, 40, 225-240

Fernandez-Duque, D., \& Thornton, I. M. (2000). Change detection without awareness: Do explicit reports underestimate the representation of change in the visual system? Visual Cognition, 7, 324-344.

Grimes, J. (1996). On the failure to detect changes in scenes across saccades. In K. A. Akins (Ed.), Perception (Vancouver Studies in Cognitive Science, Vol. 5, pp. 89-110). Oxford: Oxford University Press.

Hay hoe, M. M., Bensinger, D. G., \& Ballard, D. H. (1998). Task constraints in visual working memory. Vision Research, 38, 125-137.

Henderson, J. M. (1991). Stimulus discrimination following covert attentional orienting to an exogenous cue. Journal of Experimental Psychology: Human Perception \& Performance, 17, 91-106.

Henderson, J. M. (1996). Visual attention and the attention-action interface. In K. A. Akins (Ed.), Perception (Vancouver Studies in Cognitive Science, Vol. 5, pp. 290-316). Oxford: Oxford University Press.

Henderson, J. M., \& Hollingworth, A. (1999a). High-level scene perception. Annual Review of Psychology, 50, 243-271.

Henderson, J. M., \& Hollingworth, A. (1999b). The role of fixation position in detecting scene changes across saccades. Psychological Science, 10, 438-443.

Henderson J. M., \& Hollingworth, A. (in press). Eye movements, visual memory, and scene representation. In M. A. Peterson \& G. Rhodes (Eds.), Analytic and holistic processes in the perception of faces, objects, and scenes. New York: JAI/Ablex.

Henderson, J. M., Hollingworth, A., \& Subramanian, A. N. (1999). The retention and integration of scene information across saccades: A global change blindness effect. Paper presented at the Annual Meeting of the Psychonomic Society, Los Angeles.

Henderson, J. M., McClure, K. K., Pierce, S., \& Schrock, G. (1997). Object identification without foveal vision: Evidence from an artificial scotoma paradigm. Perception \& Psychophysics, 59, 323-346.

Hollingworth, A., \& Henderson, J. M. (2000). Semantic informativeness mediates the detection of changes in natural scenes. Visual Cognition, 7, 213-235.

Hollingworth, A., Williams, C. C., \& Henderson, J. M. (in press). To see and remember: Visually specific information is retained in memory from previously attended objects in natural scenes. Psychonomic Bulletin \& Review.

JONIDES, J. (1981). Voluntary versus automatic control over the mind's eye's movement. In J. B. Long \& A. D. Baddeley (Eds.), Attention and performance IX (pp. 187-203). Hillsdale, NJ: Erlbaum.

McConkie, G. W., \& Currie, C. B. (1996). Visual stability while viewing complex pictures. Journal of Experimental Psychology: Human Perception \& Performance, 22, 563-581.

O'Regan, J. K., Deubel, H., Clark, J. J., \& Rensink, R. A. (2000). Picture changes during blinks: Looking without seeing and seeing without looking. Visual Cognition, 7, 191-212.
Posner, M. I., Snyder, C. R., \& Davidson, B. J. (1980). Attention and the detection of signals. Journal of Experimental Psychology: General, 109, 160-174.

Rensink, R. A. (2000a). The dynamic representation of scenes. Visual Cognition, 7, 17-42.

RENSINK, R. A. (2000b). Visual search for change: A probe into the nature of attentional processing. Visual Cognition, 7, 345-376.

Rensink, R. A., O'Regan, J. K., \& Clark, J. J. (1997). To see or not to see: The need for attention to perceive changes in scenes. Psychological Science, 8, 368-373.

SCHOLL, B. J. (2000). Attenuated change blindness for exogenously attended items in a flicker paradigm. Visual Cognition, 7, 377-396.

Simons, D. J., \& Levin, D. T. (1997). Change blindness. Trends in Cognitive Sciences, 1, 261-267.

Theeuwes, J. (1991). Cross-dimensional perceptual selectivity. Perception \& Psychophysics, 50, 184-193.

Williams, P., \& Simons, D. J. (2000). Detecting changes in novel 3D objects: Effects of change magnitude, spatiotemporal continuity, and stimulus familiarity. Visual Cognition, 7, 297-322.

Wolfe, J. M. (1999). Inattentional amnesia. In V. Coltheart (Ed.), Fleeting memories (pp. 71-94). Cambridge, MA: MIT Press.

\section{NOTES}

1. One concern with this design is that the difference between the time-out duration for the change conditions and that for the catch trials could have been informative had changes often been detected after more than $20 \mathrm{sec}$ of viewing. That is, participants could have learned that if the scene was still displayed after more than $20 \mathrm{sec}$, a change had to be occurring. However, $98.9 \%$ of detections in the change conditions occurred in less than $20 \mathrm{sec}$, so the difference in time-out duration was not critical.

2. This set of stimuli was originally developed for another study (Henderson \& Hollingworth, 1999b), and we wished to retain the ability to compare results across studies, necessitating an uneven assignment of items to conditions.

3. Three of the eight participants appeared to adopt a strategy by which they stared at the center of the screen and monitored for changes in the periphery. These participants applied the strategy across multiple trials. However, they were able to detect a change without leaving the center of the screen on only a small number of trials. In these trials, the changes were particularly salient. For more difficult changes (and particularly for rotation changes), these participants were forced to leave the central region of the scene and fixate potential change targets.

4. One item was eliminated from the items analysis, owing to an empty cell in one of the change conditions.

5. Eight Michigan State University students from the same pool as in the main experiment participated. The scene stimuli, apparatus, and procedure were identical to those in the main experiment, except that on $50 \%$ of the trials, no change occurred. The remaining trials were divided between deletion and rotation changes. Across participants, each scene appeared in each condition an equal number of times.

(Manuscript received May 3, 1999; revision accepted for publication April 13, 2000.) 